\title{
Efecto de la Incorporación de Pequeñas Dosis de Emulsión Asfáltica en el Comportamiento de Estabilizaciones de Suelo con Cemento Portland
}

\section{Effect of the incorporation of small doses of emulsified asphalt in the performance of portland cement soil stabilizations}

Presentación: 10/10/2019

Aprobación: 12/08/2020

\section{Carlos Defagot}

Centro de Investigación y Desarrollo para la Construcción y la Vivienda CECOVI - Facultad Regional Santa Fe, Universidad Tecnológica Nacional - Argentina

cdefagot@frsf.utn.edu.ar

\section{Néstor Ulibarrie}

Centro de Investigación y Desarrollo para la Construcción y la Vivienda CECOVI - Facultad Regional Santa Fe, Universidad Tecnológica Nacional - Argentina

nulibarr@frsf.utn.edu.ar

\section{Pablo Hillar}

Centro de Investigación y Desarrollo para la Construcción y la Vivienda CECOVI - Facultad Regional Santa Fe, Universidad Tecnológica Nacional - Argentina

ingphillar@gmail.com

\section{RESUMEN}

La estabilización de suelos con cemento es uno de los procesos más eficientes para conformar capas portantes para estructuras de pavimento aunque simultáneamente posee una consabida debilidad en términos de su tendencia a la fisuración debido a los procesos de retracción. En el presente trabajo se incursiona en el uso de pequeñas dosis de emulsión asfáltica como complemento del cemento en la estabilización de mezclas de suelo-arena. El objetivo es el de evaluar un potencial efecto de mejora tanto en términos portantes, especialmente ante los efectos del agua, como en términos de retracción. A tal fin se estudia la respuesta portante del material, a partir de ensayos de resistencia a la compresión simple y tracción indirecta, así como la respuesta en términos de durabilidad por humedecimiento y 
secado, y en términos de retracción para lo cual se desarrolla un procedimiento de ensayo especifico.

Palabras Clave: Cemento, Emulsión, Compresión, Inmersión, Retracción

\begin{abstract}
Portland cement soil stabilizations are one of the most efficient and cost-effective ways to perform pavement structural layers, mainly when they are subjected to water aggressive environments. At the same time, and as any compromise solution, the weakness of the use of cement as a stabilizing agent resides in the shrinkage that implies an inevitable cracking of the resulting mixtures. The present work is about to analize the potential effect of the using of small doses of emulsified asphalt as a complement in the cement stabilization of sand-soil mixtures with the purpose of evaluate possible improvements in the strength behavior, even after water inmersion, and on the shrinkage level. To that end it is studied the bearing behavior of the mixtures from several tests as Unconfined compressive strength and indirect tensile strength, as the performance in terms of durability by wet-dry test and shrinkage behavior for wich is developed an specific test procedure.
\end{abstract}

Keywords: Cement, Emulsion, Compressive, Inmersion, Shrinkage

\title{
1. INTRODUCCIÓN
}

En determinados ámbitos geográficos y climáticos el uso del cemento como agente estabilizador de suelos conforma una de las maneras más eficientes y económicas de alcanzar resultados razonablemente satisfactorios. En la zona litoral, y más específicamente en la provincia de Santa Fe, donde el uso de agregados pétreos implica elevados costos de transporte y donde la agresividad climática en términos de precipitaciones, sumado a potenciales déficits de escurrimiento y altas probabilidades de anegamientos y por supuesto a la presencia de suelos de mediocre a mala calidad para el uso vial, el uso del cemento como agente estabilizador de materiales autóctonos implica soluciones eficientes a costos razonables en términos de incremento de capacidad portante y reducción de la permeabilidad.

Simultáneamente, y como toda solución de compromiso, el flanco débil del cemento como agente estabilizador es el efecto de retracción consecuente de los procesos de hidratación y fraguado que se traducen en una inevitable fisuración de las mezclas resultantes. Esta última condición implica la necesidad de acudir a un control estricto de las resistencias que puedan alcanzarse en estas mezclas a fin de disponer de una razonable probabilidad de que el material resultante no tenderá hacia un comportamiento "tipo losa", entendiéndose por ello a un patrón de fisuración transversal sistemático que transforme a la capa en un conjunto de paños sucesivos, sino mas bien hacia un proceso de microfisuración, con el fin de evitar los fenómenos de reflexión de fisuras.

La posibilidad del uso de materiales bituminosos en conjunción con el cemento, ha tenido cierto abordaje principalmente en el ámbito de los reciclados, con los llamados reciclados mixtos (Bardesi Orue-Echeverria, 1998), en los que la relación porcentual en peso entre ambos ligantes oscila proporciones de entre el 40 y $60 \%$ entre uno y otro. Otra variante de uso de esta combinación es la de la incorporación de pequeñas cantidades de cemento portland (inferiores al 1\%) sobre reciclados con emulsión asfáltica a fin de reducir la susceptibilidad 
de los mismos ante los efectos del agua sin sacrificar sus propiedades de flexibilidad (Potti, Martínez, \& Mancebo, 2002). A diferencia de los casos mencionados, en el presente estudio lo que se pretende es la incorporación de proporciones reducidas de emulsión asfáltica a mezclas cementadas, atendiendo a cuestiones de relación beneficio-costo, a fin de evaluar si dicha incorporación habría de implicar una reducción del efecto retractivo, con un incremento de la capacidad portante o al menos sin una pérdida de la misma, en especial bajo condiciones agresivas del agua.

\section{MATERIALES}

El presente trabajo ha sido desarrollado con una mezcla de suelo, arena silícea redondeada de Rio Paraná, y Cemento Portland Normal. Se ha seleccionado como referencia este tipo de material por tratarse de una mezcla típica dentro de las posibles soluciones aplicables en el ámbito vial dentro de la región Litoral, más específicamente en la Provincia de Santa Fé.

\subsection{Materiales}

A los fines de la definición de la mezcla de referencia y de la posterior incorporación de emulsión asfáltica, se utilizaron los siguientes materiales:

- Suelo Seleccionado con las características indicadas en la Tabla 1.

\begin{tabular}{|c|c|c|c|c|c|c|c|c|}
\hline \multicolumn{4}{|c|}{ Granulometría \%Pasante } & \multicolumn{3}{c|}{ Consistencia } & HRB \\
\hline \#200 & $\# 40$ & $\# 10$ & $\# 4$ & LL & LP & IP & Grupo & IG \\
\hline $\mathbf{7 2 , 5}$ & $\mathbf{9 9 , 8}$ & 100,0 & 100,0 & 34 & 20 & 15 & A6 & 9 \\
\hline
\end{tabular}

Tabla 1. Características físicas del Suelo Seleccionado

- Arena silícea de Rio Paraná con las características indicadas en la Tabla 2.

\begin{tabular}{|c|c|c|c|c|c|c|c|c|}
\hline \multicolumn{4}{|c|}{ Granulometría \%Pasante } & \multicolumn{4}{c|}{ Consistencia } & HRB \\
\hline \#200 & $\# 40$ & $\# 10$ & $\# 4$ & LL & LP & IP & Grupo & IG \\
\hline 0,3 & $\mathbf{8 9 , 0}$ & $\mathbf{9 9 , 8}$ & 100,0 & - & - & NP & A3 & 0 \\
\hline
\end{tabular}

Tabla 2. Características físicas de la Arena

(LL es Límite Liquido - LP es Límite Plástico - IP es Indice Plástico - IG es Indice de Grupo)

- Cemento Portland Normal CPN40

- Emulsión asfáltica catiónica de corte superestable según Norma IRAM 6691

\subsection{Mezcla de Suelo Arena Cemento}

La mezcla de áridos ha sido definida como una constante tomando como referencia los dosajes típicos de las mezclas de Suelo Arena Cemento que responden por lo general a un $70 \%$ de arena y un 30\% de suelo en peso seco. El resultado obtenido es suelo tipo A2-4 según la identificación HRB.

Para la determinación de las cantidades de cemento, se han definido las siguientes pautas: 
- Que la densidad de la mezcla estabilizada será del 100\% del Proctor Standard o T99

- Que el orden de magnitud de la resistencia a la compresión simple a los 7 días sea aquel que implique una elevada probabilidad de que el material resultante no tenga comportamientos tipo losa. Para ello se toma como referencia un valor portante inferior a $25 \mathrm{~kg} / \mathrm{cm} 2$ a los 7 días.

A partir de pruebas realizadas, se definió como proporción adecuada de cemento portland a un $6 \%$ en peso, la cual se tradujo en valores de la resistencia a la compresión simple del orden de los $20 \mathrm{~kg} / \mathrm{cm} 2$ a los 7 días.

\section{METODOLOGIA}

Las etapas seguidas fueron las siguientes:

- Definición de la proporción de cemento.

- Definición de los porcentajes de emulsión asfáltica

- Ensayos portantes. Resistencia a la compresión simple y Tracción indirecta

- Ensayo de durabilidad. Humedecimiento y secado

- Ensayo de retracción.

A fines de llevar adelante los procedimientos de laboratorio tendientes a la caracterización del material en estudio mediante los ensayos de resistencia a la compresión simple, tracción indirecta, durabilidad y retracción, en primer término se definieron las proporciones de emulsión asfáltica a incorporar a la mezcla de suelo arena con $6 \%$ de cemento en peso. En tal sentido se establecieron porcentajes de un 1,5\% y un 2,5\% de emsulsión asfáltica en peso, con la finalidad de evaluar potenciales modificaciones en el comportamiento del material, con un costo relativamente bajo. De esta manera los ensayos de performance se realizaron para las dosificaciones combinadas que se muestran en la Tabla 3.

\begin{tabular}{|c|c|}
\hline \multirow{2}{*}{ Mezcla } & Emulsión \\
\cline { 2 - 2 } & $\%$ \\
\hline 2 & 0,0 \\
\hline 3 & 1,5 \\
\hline
\end{tabular}

Tabla 3. Dosificaciones de Emulsión Asfáltica utilizadas

Respecto a las condiciones de ensayo referentes a la resistencia a la compresión simple, tracción indirecta y durabilidad, se tuvo especial precaución en que las mismas permitiesen valorar de manera adecuada el efecto resistente de la emulsión asfáltica. Para ello, después de analizar diversas alternativas, se consideró como la más razonable, aquella en que la mezcla de suelo arena y cemento con incorporación de emulsión asfáltica alcanzase una humedad residual del orden del $60 \%$ de la humedad de moldeo. Esta última condición es la que usualmente se considera al momento de evaluar estabilizaciones de suelos con emulsión asfáltica a fin de que las mismas arriben a una condición de equilibrio en términos de humedad residual (Petroni, Poncino, \& Cassan, 1990). Para alcanzar esta condición todas 
las probetas moldeadas fueron sometidas a 7 días de curado, y luego a reposo a temperatura ambiente hasta alcanzar la humedad de referencia. Este tiempo adicional fue de 10 días para todos los casos. De esta manera la edad de todas las probetas ensayadas (con y sin emulsión asfáltica) fue de 17días. La condición referida no fue aplicada al caso de los ensayos de retracción, pues el objetivo en ese caso fue evaluar la respuesta de las mezclas en las edades más tempranas del proceso.

\subsection{Efecto agresivo del agua}

Se consideró para los casos de respuesta portante, el análisis de alternativas sometidas a efectos agresivos del agua. Para evaluar esta condición las probetas correspondientes fueron sometidas a una inmersión en agua a temperatura ambiente durante 48 hs para su posterior rotura. De esta manera se dispusieron de valores portantes del material sin inmersión y con inmersión de 48 hs, a fin de cuantificar la condición de resistencia conservada. El concepto de resistencia conservada debe interpretarse como la relación porcentual entre la resistencia a la compresión simple después de la inmersión y la resistencia a la compresión simple sin inmersión.

\subsection{Condiciones de moldeo}

Las probetas utilizadas para los ensayos de rotura a la compresión simple, tracción indirecta y durabilidad por humedecimiento y secado se moldearon mediante ensayo proctor T99 molde chico, con una humedad del 12\%, a partir de lo establecido en la Norma VN-E1966 "Compactación de mezclas de suelo-cemento y suelo-cal”. Las probetas utilizadas para el ensayo de retracción fueron moldeadas siguiendo un procedimiento específico detallado en el apartado 3.6"Retracción", bajo la premisa de conservar la misma energía de compactación que en los restantes casos.

En lo que respecta al moldeo de las probetas para evaluar la retracción del material, ante la necesidad de disponer de una longitud de medición efectiva de $30 \mathrm{~cm}$ a fin de poder utilizar el comparador de longitud portátil y de apreciación milesimal disponible se decidió emplear un molde ad-hoc confeccionado de PVC de $\varnothing 110 \mathrm{~mm}$ (diámetro del molde Proctor chico), cortado en dos directrices opuestas (materializando dos medias cañas) para facilitar el desmolde (manteniendo al momento del moldeo, el molde cerrado con abrazaderas metálicas). De esta manera fue posible conformar probetas de un diámetro similar al de los moldes Proctor pero con una longitud de $30 \mathrm{~cm}$. Se cuidó específicamente de mantener la energía de compactación asociada al Proctor Standard.

En lo referente a la incidencia de la temperatura, parámetro que importa por las características termodependientes de los asfaltos, se consideró neutralizado su efecto al momento de optar por moldear la totalidad de las probetas en simultáneo,para cada tipo de ensayo de modo de mantener un historial térmico homogéneo. Adicionalmente se consideró razonable la evaluación de la respuesta de los materiales a la condición de "temperatura ambiente" como medida representativa de un potencial comportamiento real de los mismos.

\subsection{Resistencia a la Compresión Simple}

Se siguió el proceso establecido en la Norma VN-E33-67 "Ensayo de compresión de probetas compactadas desuelo-cal y suelo-cemento", modificado según lo referido previamente en relación al periodo de reposo post-curado y a las condiciones adicionales de inmersión previstas a fin de evaluar la resistencia conservada. Los ensayos de resistencia a la compresión simple se realizaron con las probetas bajo una temperatura ambiente de $16^{\circ} \mathrm{C}$. 


\subsection{Resistencia a la Tracción Indirecta}

En virtud de la falta de una normativa específica de referencia para la rotura de probetas de suelo cemento a la tracción indirecta por compresión diametral, se tomó como referencia la Norma IRAM 1658:1995 "Hormigón. Determinación de la resistencia a la tracción simple porcompresión diametral”. Partiendo de que las diferencias relevantes entre el ensayo de resistencia a la compresión simple de probetas de suelo cemento (Norma VN-E33-67) y el de probetas de Hormigón (Norma IRAM 1546:2013 "Hormigón de cemento portland. Método de ensayo a compresión”) están referidas a la esbeltez de las probetas, y que por otro lado dichas condiciones de esbeltez son similares, tanto para el ensayo de rotura a la compresión simple (Norma IRAM 1546:2013) como para el ensayo de tracción indirecta de probetas de hormigón(Norma IRAM 1658:1995), se ha considerado coherente mantener la esbeltez prevista en la Norma VN-E33-67, así como las condiciones de carga también previstas en la misma, al momento de evaluar la tracción indirecta en las probetas de suelo arena cemento.

En la Figura 1 se muestran dos imágenes; en la de la izquierda la probeta está ubicada en el dispositivo de ensayo y en de la derecha, la probeta ya está ensayada.

Las condiciones referidas a los periodos de curado y reposo, así como de inmersión, son las mismas que las establecidas para el ensayo de resistencia a la compresión simple. Los ensayos de resistencia a la tracción indirecta se realizaron con las probetas bajo una temperatura ambiente de $17^{\circ} \mathrm{C}$.

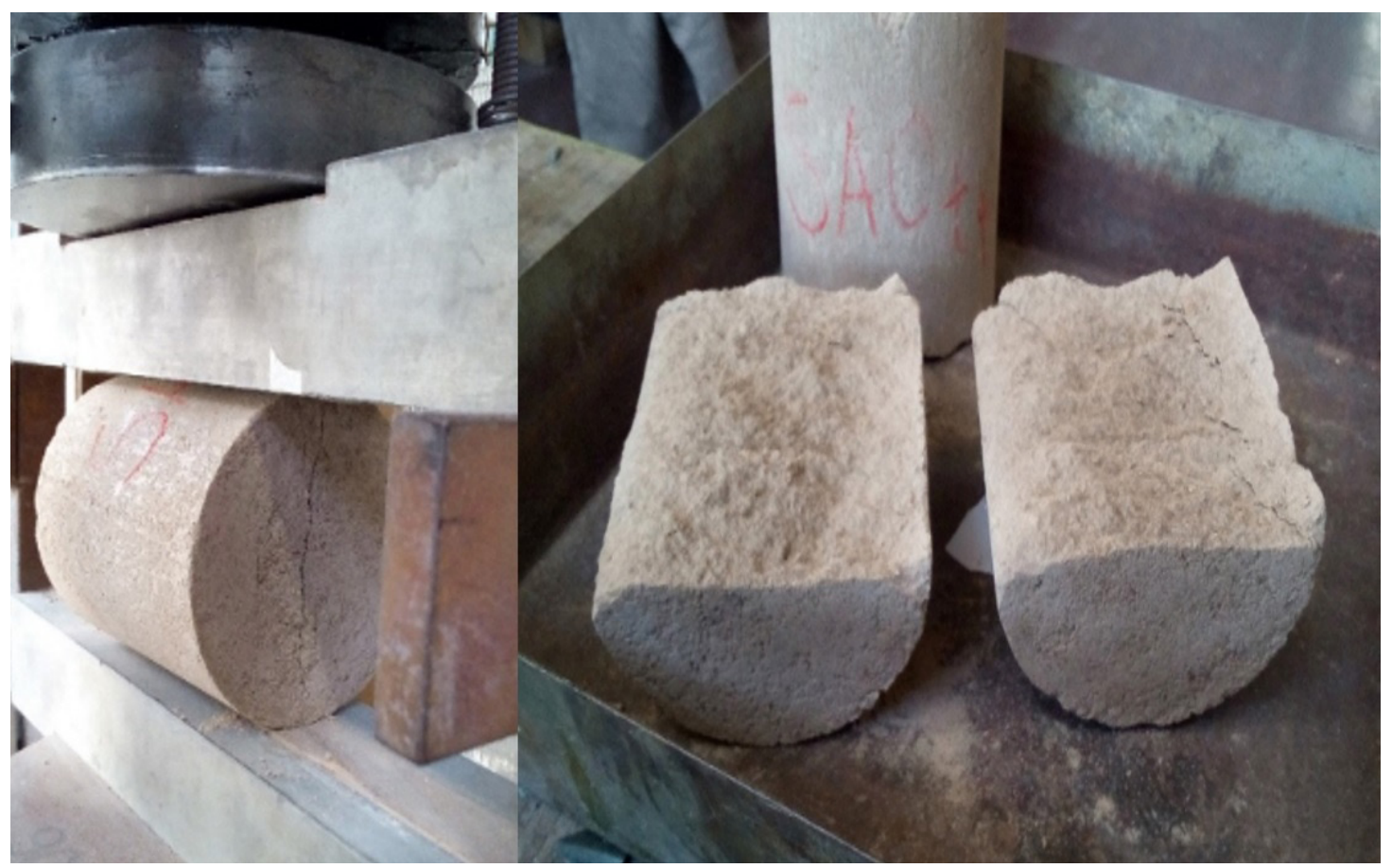

Figura 1. Ensayo y rotura de probetas a la tracción indirecta por compresión diametral

\subsection{Durabilidad por humedecimiento y secado}

Se siguió el proceso establecido en la Norma VN-E21-66 "Ensayo de durabilidad por humedecimiento y secado de mezclas de suelo-cemento”, modificado según lo referido 
previamente en relación al periodo de reposo post-curado a fin de disponer de las mismas condiciones utilizadas para el resto de los ensayos. Mediante el ensayo de referencia se pretende valorar la incidencia de la incorporación de la emulsión asfáltica en la respuesta de la mezcla ante un entorno agresivo definido por la combinación de los 12 ciclos de humedecimiento y secado y desgaste por cepillado previstos en la normativa. En este caso los resultados se expresan como la relación porcentual entre la pérdida de peso acumulada experimentada por el material respecto al peso inicial de la muestra de ensayo.

\subsection{Retracción}

El objeto de este análisis fue determinar la magnitud de la retracción volumétrica de las probetas moldeadas a fin de inferir potenciales diferencias entre la respuesta del suelo arena cemento, y del suelo arena cemento con incorporación de emulsión asfáltica. Para llevar a cabo este análisis, se diseñó una metodología consistente en medir variaciones de longitud de un segmento de definición previa. A partir de esta referencia y mediante sucesivas mediciones, es posible determinar la variación entre lecturas consecutivas por diferencia entre las mismas, la cual estará asociada además a un determinado período de tiempo. Para ello se utilizó un comparador de longitudes con una apreciación de 0,001 mm, aplicado sobre puntos de control materializados por medio de placas botón de aluminio adheridas (con resina epoxi) en la cara de la probeta, en una directriz definida previamente. La distancia entre los centros de esas placas (donde se aplica cada punto de apoyo del comparador) constituye el segmento que es monitoreado con las mediciones sucesivas. Para medir la variación de longitud de la probeta se eligieron dos directrices opuestas de modo de determinar en el promedio la evolución de deformación axial de la probeta llevada al eje de la misma. La frecuencia de medición fue determinada cada 24 hs, siendo relevante la magnitud acumulada de la retracción al cabo de los 7 días del período de curado (Figura 2).
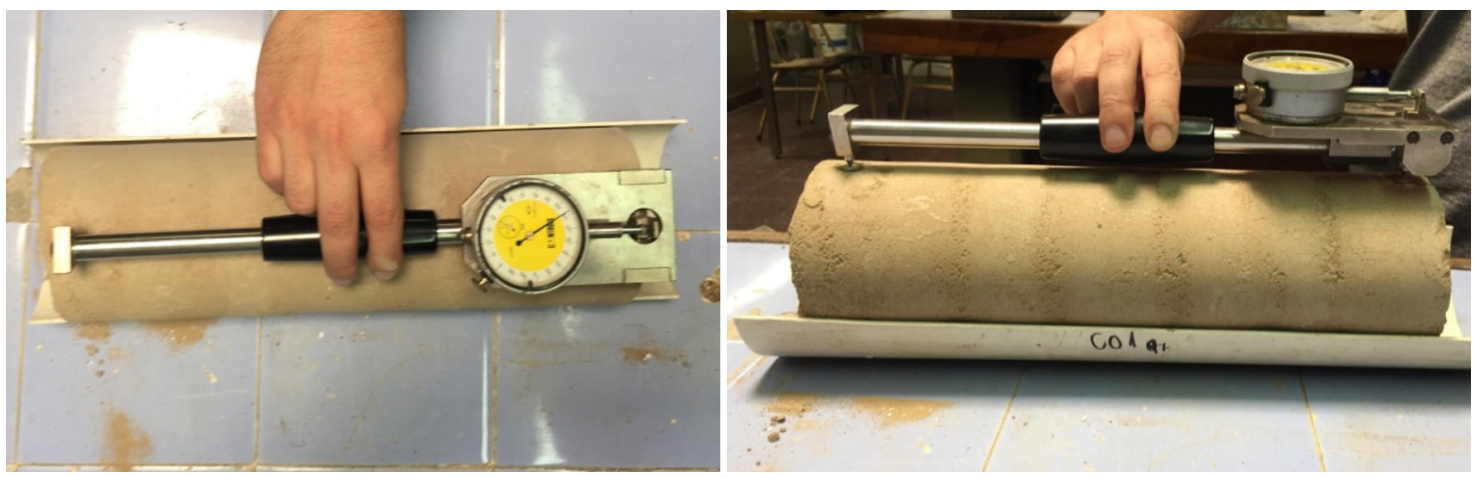

Figura 2. Medición de la variación de longitud debida a la retracción

\section{RESULTADOS Y DISCUSION}

\subsection{Efectos en la Resistencia a la Compresión Simple}

En la Tabla 4 se presentan los resultados de la resistencia a compresión simple; la incorporación de proporciones reducidas de emulsión asfáltica a las mezclas de suelo arena cemento utilizadas, no se tradujo en tendencias relevantes en cuanto a la relación entre 
la resistencia a la compresión simple pre y post-inmersión (resistencia conservada), advirtiéndose además un leve incremento de la dispersión en esta relación con el incremento de emulsión asfáltica.

\begin{tabular}{|c|c|c|c|}
\hline \multirow{2}{*}{ Mezcla } & \multicolumn{2}{|c|}{ RCS promedio $(\mathrm{kg} / \mathrm{cm} 2)$} & Entorno \\
& Sin inmersión & Con inmersión & Conservada (\%) \\
\hline 1 & 29,0 & 16,5 & 55 a 58 \\
\hline 2 & 27,0 & 16,0 & 53 a 63 \\
\hline 3 & 24,5 & 14,0 & 48 a 65 \\
\hline
\end{tabular}

Tabla 4. Resistencia a la compresión simple

Esta tendencia en los resultados ha podido advertirse en otros casos estudiados de acuerdo a la bibliografía asumida como referencia, aunque bajo diferentes características de los materiales. Por ejemplo en el trabajo "Características mecánicas de las mezclas grava-cemento-emulsión. Influencia de la composición” (Raz \& Gordillo Gracia, 1998), si bien el mismo se referencia en materiales granulares, es posible observar como a partir de determinados incrementos en la proporción de cemento respecto de la de emulsión asfáltica, las resistencias conservadas tienden a decrecer con el incremento de la cantidad de emulsión asfáltica. En otro orden de análisis en el trabajo "Evaluation of for road pavements" (Baghini, Amiruddin , \& Bin Karim, 2015), se concluye a partir del estudio de mezclas granulares con cemento y emulsión asfáltica (en proporciones medianamente del mismo orden) que existe una tendencia a la baja en la resistencia a la compresión simple en seco a partir de determinada cantidad de emulsión asfáltica. En este último caso los autores se inclinan por explicar el cambio de tendencia de la resistencia en función de la proporción de emulsión en una incidencia de la magnitud de esta en las relaciones humedad-densidad del material.En el presente estudio, cuestiones de esta índole fueron tenidas en cuenta al momento de definir las pautas y criterios asumidos para el curado e inmersión de las probetas antes referidas. Adicionalmente la premisa fue siempre considerar el agua de la emulsión asfáltica como parte de la humedad de moldeo.

\subsection{Efectos en la Resistencia a la Tracción Indirecta}

En lo referente a la respuesta a la tracción, en la Tabla 5 se observa una tendencia de mejora en la resistencia conservada a partir de la incorporación de pequeñas dosis de emulsión asfáltica a las mezclas de suelo arena cemento utilizadas. Esta mejora se mostró más relevante en la mezcla 2, con la incorporación del 1,5\% de emulsión asfáltica. La mejora mencionada no solo fue advertida en la resistencia conservada, sino también en los valores medios absolutos de la resistencia a la tracción indirecta.

\begin{tabular}{|c|c|c|c|}
\hline \multirow{2}{*}{ Mezcla } & \multicolumn{2}{|c|}{ RTI promedio $(\mathrm{kg} / \mathrm{cm} 2)$} & Entorno \\
& Sin inmersión & Con inmersión & Conservada (\%) \\
\hline 1 & 5,8 & 3,6 & 60 a 65 \\
\hline 2 & 6,0 & 4,2 & 68 a 76 \\
\hline 3 & 5,3 & 3,5 & 65 a 68 \\
\hline
\end{tabular}

Tabla 5. Resistencia a la tracción indirecta 
Adicionalmente se ha considerado razonable la relación obtenida entre la Resistencia a la tracción y la resistencia a la compresión, si se toman como referencia trabajos previamente realizados, donde para mezclas similares a las estudiadas la relación mencionada es del orden del 19\% (Poncino, Corini, \& Cassan, 1987). En lo referente a mezclas de suelos estabilizados con emulsión asfáltica (Suelo Arena Emulsión), se dispone de antecedentes con una relación sensiblemente inferior, con relaciones entre resistencia a la tracción y a la compresión de entre el 10 y el 13\% para probetas moldeadas en laboratorio (Ermáccora, Hillar, Zocco, \& Diez, 2000). En el presente estudio, y en términos de relación de promedios, la relación obtenida fue del 20\% para las probetas sin emulsión asfáltica y del 22\% para las con emulsión asfáltica sin inmersión. Bajo la condición de inmersión las diferencias se incrementaron aun algo mas, siendo del $22 \%$ para el caso sin emulsión asfáltica y del 26 y $25 \%$ para el caso con emulsión asfáltica. Estas comparaciones ponen de manifiesto un aspecto más de los términos de mejora de comportamiento a la tracción experimentados a partir de la incorporación del material bituminoso, como se observa en la Figura 3.

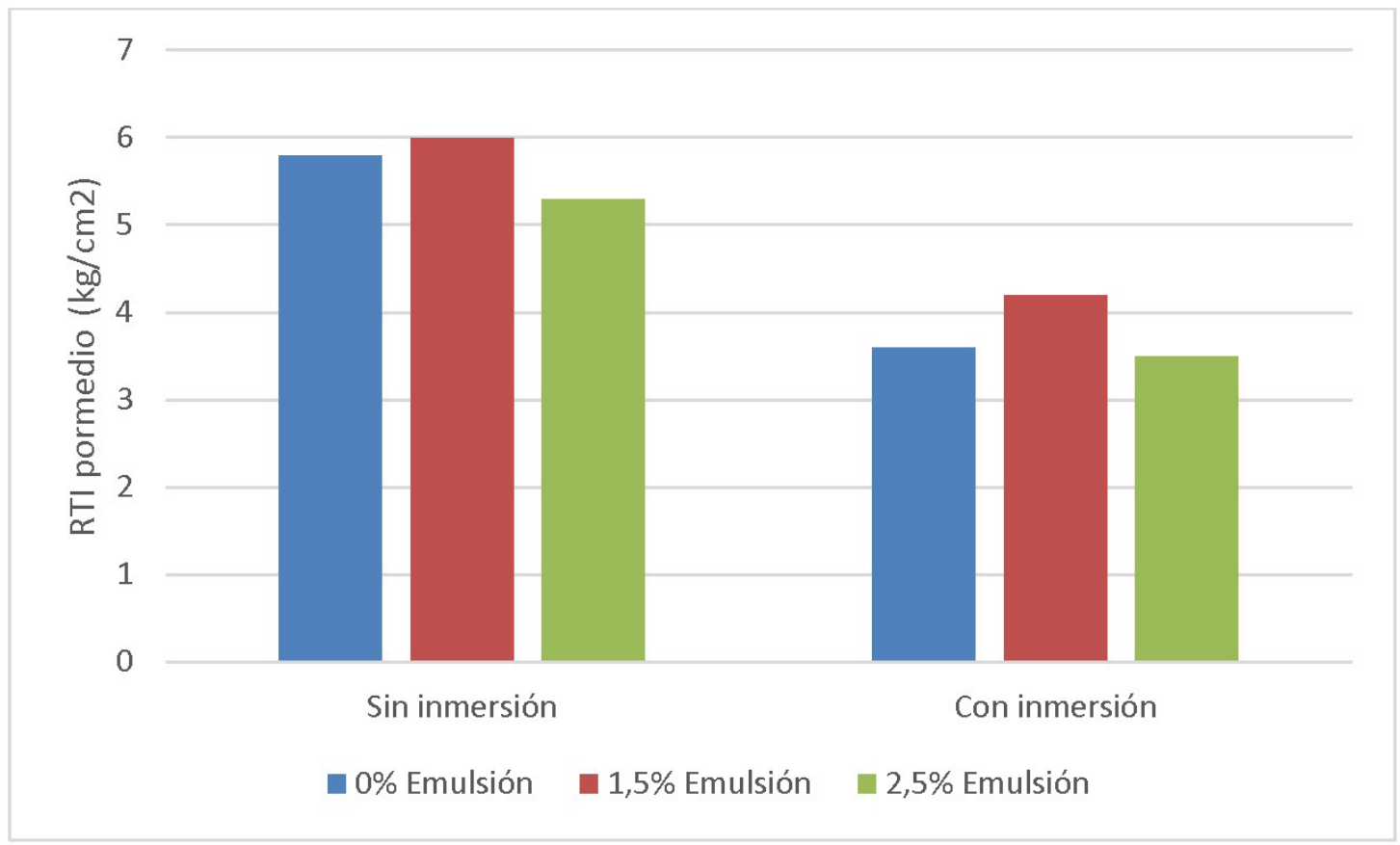

Figura 3. Resultados comparativos de Resistencia a la Tracción Indirecta

\subsection{Efectos en la Durabilidad por Humedecimiento y Secado}

En el caso de los ensayos de durabilidad por humedecimiento y secado, la incorporación de las dosis de emulsión referidas no tuvo impacto positivo en la respuesta de las probetas ensayadas, denotando además una respuesta inferior a partir de la incorporación de emulsión asfáltica, marcadamente mayor con el tenor de 2,5\% de emulsión, como puede observarse en la Tabla 6. 


\begin{tabular}{|c|c|c|c|}
\hline \multirow{2}{*}{ Mezcla } & Emulsión & \multicolumn{2}{|c|}{ Pérdida de peso (\%) } \\
\cline { 2 - 4 } & $\%$ & Sin cepillado & Con Cepillado \\
\hline 1 & 0,0 & 8,0 & 20,1 \\
\hline 2 & 1,5 & 8,6 & 26,2 \\
\hline 3 & 2,5 & 12,6 & 23,8 \\
\hline
\end{tabular}

Tabla 6. Pérdida por Humedecimiento y Secado

Los resultados obtenidos no deberían asociarse en este caso al simple hecho de incorporar asfaltos a la mezcla cementada, sino más bien al hecho de que ello se ha realizado en pequeñas dosis. Probablemente una incorporación de material bituminoso en cantidades mayores, o inclusive en proporciones importantes reemplazando a parte del cemento, se traduciría en una mejora del comportamiento. Lo referido es posible de inferir a partir de los efectos impermeabilizantes de la emulsión asfáltica sobre mezclas de suelo arena (Petroni, et al., 1990).

\subsection{Efectos en la Retracción}

En lo referente al ensayo de referencia, las características del método empleado, adaptado para la ocasión, sus ajustes previos, así como diversas complicaciones y dificultades determinadas en el proceso,determinaron que se eligiera una comparación directa en el comportamiento a esta propiedad entre probetas sin emulsión y con un tenor de $1,5 \%$ de emulsión (atendiendo que esa dosificación venía mostrando mejores resultados que la otra elegida con un mayor tenor de emulsión). Los resultados de los ensayos (tomados como promedio de las distintas muestras elaboradas) se observan en la Tabla 7.

\begin{tabular}{|c|c|c|}
\hline \multirow{2}{*}{ Mezcla } & Emulsión & Retracción \\
& $\%$ & $\%$ \\
\hline 1 & 0,0 & 15,0 \\
\hline 2 & 1,5 & 14,0 \\
\hline
\end{tabular}

Tabla 7. Retracción

Si bien estos resultados obtenidos, indican una leve reducción en la retracción del material con la incorporación de una dosis reducida de emulsión asfáltica, la escasa diferencia de comportamiento medido como resultado no permite afirmar la presencia de una tendencia, siendo ello mas bien y a priori, un indicador que no se han alcanzado variaciones de relevancia. Se ha considerado este aspecto específicamente como un punto de importancia sobre el cual profundizar los estudios realizados, sumando más determinaciones para otros tenores intermedios de incorporación de emulsión asfáltica.

\section{CONCLUSIONES}

A partir de los estudios realizados y los resultados obtenidos es posible concluir que las dosis de emulsión asfáltica incorporadas a la mezcla de suelo arena cemento, han puesto de manifiesto una mejora en la respuesta del material cuando es sometido a efectos de tracción, en especial en condición post-inmersión. Esta condición de mejora no ha sido posible 
de apreciar en la respuesta a compresión de las mezclas estudiadas, donde los resultados no han marcado tendencias, especialmente en términos de resistencia conservada.

En lo referente a la respuesta por durabilidad por humedecimiento y secado los resultados obtenidos estarían indicando que las cantidades de material bituminoso incorporado son escasas para tener un impacto de relevancia en la respuesta del material ante el contexto agresivo previsto por la normativa de referencia.

Con respecto a la retracción del material, la escasa reducción de la magnitud de la misma ante la incorporación de emulsión asfáltica en combinación con las dificultades encontradas en el proceso de ensayo abonan la necesidad de profundizar en este aspecto bajo la premisa de optimizar la implementación de dicho procedimiento. Esto último, atendiendo que se obtuvieron resultados para esta propiedad, a partir de un método no normalizado, adaptado de otras técnicas de ensayo parecidas que merece ajustes para poder ser replicado con facilidad en otros ámbitos.

Como líneade trabajo a futuro se propone la profundización del estudio, usando otros tenores de emulsión asfáltica, y reduciendo a su vez el de cemento, sobre todo para las propiedades que han mostrado alguna tendencia de cambio de comportamiento. 


\section{REFERENCIAS}

Baghini, M.S., Amiruddin, I. y Mohamed, R.B.K. (2015). Evaluation of cement-treated mixtures with slow setting bitumen emulsion as base course material for road pavements. Recuperado de https://tarjomefa.com/wp-content/uploads/2016/08/5103-English.pdf

Bardesi Orue-Echeverria, A (junio, 1998). Proyecto y dimensionamiento de firmes con capas recicladas. Trabajo presentado en el IV Congreso Nacional de Firmes. Reciclado de Firmes. Segovia (España).

Norma VN-E19-66 (1966) Compactación de mezclas de suelo-cemento y suelo-cal. Dirección Nacional de Vialidad

Norma VN-E21-66 (1966) Ensayo de durabilidad por humedecimiento y secado de mezclas de suelo-cemento. Dirección Nacional de Vialidad

Norma VN-E33-67 (1967) Ensayo de compresión de probetas compactadas desuelo-cal y suelo-cemento. Dirección Nacional de Vialidad

Ermáccora, E., Hillar, P., Zocco, C. y Diez, J.M. (noviembre, 2000). Estabilizados de Suelo Arena Emulsión en la provincia de Santa Fe. Trabajo presentado en la XXXI Reunión del Asfalto. Villa Carlos Paz, Argentina.

Norma IRAM 1658:1995 (1995) Hormigón. Determinación de la resistencia a la tracción simple por compresión diametral. Instituto Argentino de Normalización y Certificación.

Norma IRAM 1546:2013 (2013) Hormigón de cemento portland. Método de ensayo a compresión. Instituto Argentino de Normalización y Certificación.

Petroni, E., Poncino, H. y Cassan, R. (diciembre, 1990) Dosificación y caracterización de estabilizados de Suelo Área Emulsión Asfáltica. Trabajo presentado en la XXVI Reunión Anual del Asfalto. Buenos Aires, Argentina.

Poncino, H., Corini, G. y Cassan, R. (noviembre, 1987) Caracterización de materiales tratados con ligantes hidráulicos. Su aplicación al dimensionamiento de pavimentos flexibles. Trabajo presentado en el IV Congreso Ibero-Latinoamericano del Asfalto. Ciudad de México, México

Potti, J.J., Martínez, M. y Mancebo, J. (2002). Avances en el campo de los reciclados. Reciclado mixto con emulsión y cemento. Recuperado de http://www.crp.pt/docs/A11S96-pavimentos_25. pdf

Raz, R.T. y Gordillo Gracia, J. (junio, 1998) Características mecánicas de las mezclas grava-cemento-emulsión. Influencia de la composición. Trabajo presentado en el IV Congreso Nacional de Firmes. Reciclado de Firmes. Segovia, España. 